\title{
Report of Health Care Provider Recommendation for COVID-19 Vaccination Among Adults, by Recipient COVID-19 Vaccination Status and Attitudes - United States, April-September 2021
}

\begin{abstract}
Kimberly H. Nguyen, DrPH${ }^{1}$; David Yankey, $\mathrm{PhD}^{1}$; Peng-jun Lu, MD ${ }^{1}$; Jennifer L. Kriss, $\mathrm{PhD}^{1}$; Noel T. Brewer, PhD 2 ; Hilda Razzaghi, PhD ${ }^{1}$; Mehreen Meghani, MPH ${ }^{1}$; Brian J. Manns, PharmD ${ }^{1}$; James T. Lee, $\mathrm{MD}^{1}$; James A. Singleton, PhD ${ }^{1}$
\end{abstract}

Vaccination is critical to controlling the COVID-19 pandemic, and health care providers play an important role in achieving high vaccination coverage ( 1 ). To examine the prevalence of report of a provider recommendation for COVID-19 vaccination and its association with COVID-19 vaccination coverage and attitudes, CDC analyzed data among adults aged $\geq 18$ years from the National Immunization Survey-Adult COVID Module (NIS-ACM), a nationally representative cellular telephone survey. Prevalence of report of a provider recommendation for COVID-19 vaccination among adults increased from 34.6\%, during April 22-May 29, to 40.5\%, during August 29-September 25, 2021. Adults who reported a provider recommendation for COVID-19 vaccination were more likely to have received $\geq 1$ dose of a COVID-19 vaccine $(77.6 \%)$ than were those who did not receive a recommendation $(61.9 \%)$ (adjusted prevalence ratio $[\mathrm{aPR}]=1.12$ ). Report of a provider recommendation was associated with concern about COVID-19 (aPR = 1.31), belief that COVID-19 vaccines are important to protect oneself $(\mathrm{aPR}=1.15)$, belief that COVID-19 vaccination was very or completely safe $(\mathrm{aPR}=1.17)$, and perception that many or all of their family and friends had received COVID-19 vaccination $(\mathrm{aPR}=1.19)$. Empowering health care providers to recommend vaccination to their patients could help reinforce confidence in, and increase coverage with, COVID-19 vaccines, particularly among groups known to have lower COVID-19 vaccination coverage, including younger adults, racial/ethnic minorities, and rural residents.

NIS-ACM is a nationally representative household telephone survey of noninstitutionalized U.S. adults aged $\geq 18$ years that uses a random-digit-dialed sample of cellular telephone numbers stratified by state and selected local jurisdictions (2). Data from five data collection periods were used for these analyses: April 22-May 29, May 30-June 26, June 27-July 31, August 1-August 28, and August 29-September 25, 2021. Response rates for these five periods ranged from $17.2 \%$ to $20.9 \% *$; sample sizes ranged from 56,749 to 77,162 , with an overall sample size of 340,543 participants.

\footnotetext{
${ }^{*}$ Calculated according to the American Association for Public Opinion Research type 3 response rate. https:/www.aapor.org/AAPOR_Main/media/publications/ Standard-Definitions20169theditionfinal.pdf
}

INSIDE

1731 SARS-CoV-2 B.1.1.529 (Omicron) Variant — United States, December 1-8, 2021

1735 Booster and Additional Primary Dose COVID-19 Vaccinations Among Adults Aged $\geq 65$ Years United States, August 13, 2021-November 19, 2021

1740 Trends in and Characteristics of Drug Overdose Deaths Involving Illicitly Manufactured Fentanyls United States, 2019-2020

1747 Notes from the Field: Mucormycosis Cases During the COVID-19 Pandemic - Honduras, MaySeptember 2021

1750 Notes from the Field: COVID-19-Associated Mucormycosis - Arkansas, July-September 2021

1752 QuickStats

Continuing Education examination available at https://www.cdc.gov/mmwr/mmwr_continuingEducation.html

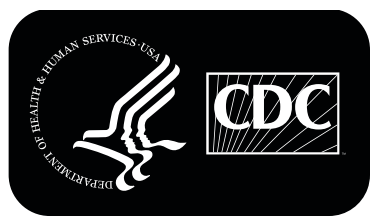


The survey assessed report of health care provider recommendation for COVID-19 vaccination, ${ }^{\dagger}$ COVID-19 vaccination status, ${ }^{\S}$ and attitudes toward vaccination. Attitudes toward vaccination were assessed by responses to four questions regarding 1) concern about COVID-19 infection (risk appraisal), 2) belief about the importance of COVID-19 vaccination (confidence), 3) belief about the safety of COVID-19 vaccination (confidence), and 4) belief about how many family and friends had received COVID-19 vaccination (social norms). These questions are based on the Behavioral and Social Drivers framework for increasing vaccine confidence $(1)$.

Prevalence of report of provider recommendation was assessed during April 22-September 25, 2021, and by period of data collection, sociodemographic characteristics, ${ }^{* *}$ U.S. Department of Health and Human Services (HHS) region, ${ }^{\dagger \dagger}$ and jurisdiction. ${ }^{\$}$ Logistic regression was used to generate unadjusted and adjusted prevalence ratios (PRs and aPRs) of the association between the four attitudinal measures and both provider recommendation for COVID-19 vaccination and COVID-19 vaccination status. Adjusted analyses controlled for age group, sex, transgender identity, sexual orientation, race/ ethnicity, education, income, insurance status, metropolitan

\footnotetext{
$\dagger$ Report of a provider recommendation was assessed with the following question: "Has a doctor, nurse, or other health professional ever recommended that you get a COVID-19 vaccine?"

$\$$ COVID-19 vaccination status was assessed by response to the following question: "Have you received at least one dose of a COVID-19 vaccine?"
}

\begin{abstract}
9 Concern about COVID-19 infection was assessed by the following questions: "How concerned are you about getting COVID-19? Would you say you are: not at all concerned; a little concerned; moderately concerned; or very concerned?" Adults who responded with moderately or very concerned were categorized as being concerned about COVID-19 infection. Beliefs about the importance of vaccination was assessed by the following question: "How important do you think getting a COVID-19 vaccine is to protect yourself against COVID-19? Would you say it is not at all important, a little important, somewhat important, or very important?" Adults who responded with somewhat or very important were categorized as believing that vaccination is important for protection against COVID-19. Beliefs about the safety of COVID-19 vaccines was assessed by the following questions: "How safe do you think a COVID-19 vaccine is for you? Would you say not at all safe; somewhat safe; very safe; or completely safe?" Adults who responded with very or completely safe were categorized as having beliefs that vaccine is safe. Finally, social norms were assessed by the following questions: "If you had to guess, about how many of your family and friends have received a COVID-19 vaccine? Would you say none; some; many; or almost all?" Adults who responded with many or almost all were categorized as having family and friends who were all or mostly vaccinated.

** Sociodemographic demographic characteristics were age group, sex, transgender identity, sexual orientation, race/ethnicity, education, income, insurance status, MSA, U.S. Census region, comorbidity status, disability status, essential worker status, and work or school requirement.

t† Region 1: Connecticut, Maine, Massachusetts, New Hampshire, Rhode Island, and Vermont; Region 2: New Jersey, New York, Puerto Rico, and the U.S. Virgin Islands; Region 3: Delaware, District of Columbia, Maryland, Pennsylvania, Virginia, and West Virginia; Region 4: Alabama, Florida, Georgia, Kentucky, Mississippi, North Carolina, South Carolina, and Tennessee); Region 5: Illinois, Indiana, Michigan, Minnesota, Ohio, and Wisconsin; Region 6: Arkansas, Louisiana, New Mexico, Oklahoma, and Texas; Region 7: Iowa, Kansas, Missouri, and Nebraska; Region 8: Colorado, Montana, North Dakota, South Dakota, Utah, and Wyoming; Region 9: Arizona, California, Hawaii, Nevada, American Samoa, Northern Mariana Islands, Federated States of Micronesia, Guam, Marshall Islands, and Palau; Region 10: Alaska, Idaho, Oregon, and Washington.
\end{abstract}

The MMWR series of publications is published by the Center for Surveillance, Epidemiology, and Laboratory Services, Centers for Disease Control and Prevention (CDC), U.S. Department of Health and Human Services, Atlanta, GA 30329-4027.

Suggested citation: [Author names; first three, then et al., if more than six.] [Report title]. MMWR Morb Mortal Wkly Rep 2021;70:[inclusive page numbers].

\author{
Centers for Disease Control and Prevention \\ Rochelle P. Walensky, MD, MPH, Director \\ Debra Houry, MD, MPH, Acting Principal Deputy Director \\ Daniel B. Jernigan, MD, MPH, Deputy Director for Public Health Science and Surveillance \\ Rebecca Bunnell, PhD, MEd, Director, Office of Science \\ Jennifer Layden, MD, PhD, Deputy Director, Office of Science \\ Michael F. Iademarco, MD, MPH, Director, Center for Surveillance, Epidemiology, and Laboratory Services
}

MMWR Editorial and Production Staff (Weekly)

Charlotte K. Kent, $\mathrm{PhD}$, MPH, Editor in Chief Jacqueline Gindler, MD, Editor

Brian A. King, PhD, MPH, Guest Science Editor

Paul Z. Siegel, MD, MPH, Associate Editor

Mary Dott, MD, MPH, Online Editor

Terisa F. Rutledge, Managing Editor

Teresa M. Hood, MS, Lead Technical Writer-Editor

Leigh Berdon, Glenn Damon, Soumya Dunworth, PhD,

Tiana Garrett-Cherry, PhD, MPH, Srila Sen, MA

Stacy Simon, MA, Morgan Thompson, Technical Writer-Editors

Matthew L. Boulton, MD, MPH

Carolyn Brooks, ScD, MA

Jay C. Butler, MD

Virginia A. Caine, MD

Jonathan E. Fielding, MD, MPH, MBA

David W. Fleming, MD
Martha F. Boyd, Lead Visual Information Specialist

Alexander J. Gottardy, Maureen A. Leahy,

Julia C. Martinroe, Stephen R. Spriggs, Tong Yang, Visual Information Specialists

Quang M. Doan, MBA, Phyllis H. King,

Terraye M. Starr, Moua Yang,

Information Technology Specialists
Ian Branam, MA,

Acting Lead Health Communication Specialist Shelton Bartley, MPH, Leslie Hamlin,

Lowery Johnson, Amanda Ray,

Health Communication Specialists

Will Yang, MA,

Visual Information Specialist

\section{MMWR Editorial Board}

Timothy F. Jones, MD, Chairman

William E. Halperin, MD, DrPH, MPH

Jewel Mullen, MD, MPH, MPA

Jeff Niederdeppe, $\mathrm{PhD}$

Celeste Philip, MD, MPH

Patricia Quinlisk, MD, MPH

Patrick L. Remington, MD, MPH
Carlos Roig, MS, MA William Schaffner, MD

Nathaniel Smith, MD, MPH

Morgan Bobb Swanson, BS

Abbigail Tumpey, MPH 
statistical area (MSA), 99 U.S. Census region, comorbidity status, ${ }^{* * *}$ disability status, ${ }^{\dagger \dagger}$ essential worker status, ${ }^{\$ \$ \$}$ and work or school COVID-19 vaccination requirement. 999 All variables assessed in this study were self-reported. The interaction between provider recommendation and each sociodemographic characteristic in predicting COVID-19 vaccination status was also assessed. The ecologic association between jurisdiction-level provider recommendation prevalence and jurisdiction-level vaccination coverage was also assessed using a Pearson correlation coefficient.

Data were analyzed using SAS (version 9.4; SAS Institute) and SUDAAN (version 11.0.3; RTI International). Results were weighted to represent the noninstitutionalized U.S. adult population aged $\geq 18$ years and calibrated to COVID-19

$\$ \$$ Fifty-three jurisdictions were defined as the 50 states; Washington, DC; and two U.S. territories (Puerto Rico and U.S. Virgin Islands). The U.S. territory of Guam was excluded because of a limited data collection period.

99 MSA status was determined based on household reported city and county of residence and was grouped into three categories: MSA principal city (urban), MSA nonprincipal city (suburban), and non-MSA (rural). MSAs and principal cities were as defined by the U.S. Census Bureau. https://www. census.gov/programs-surveys/metro-micro.html

*** Comorbidity status was ascertained by the following question: "Do you have a health condition that may put you at higher risk for COVID-19?" This was followed by the question, "Can you tell me what that is?" Responses to this second question indicate $\geq 75 \%$ of respondents interpreted the question as medical conditions that have been associated with higher risk of severe COVID-19.

$\dagger \dagger \dagger$ Disability status was ascertained by the following question: "Do you have serious difficulty seeing, hearing, walking, remembering, making decisions, or communicating?"

$\$ \mathbb{S}$ Essential worker status was defined based on the following questions: "Are you a frontline or essential worker according to your state or region?" and "In what location or setting do you currently work?" Response options were 1) health care (e.g., hospital, doctor, dentist or mental health specialist office, outpatient facility, long-term care, home health care, pharmacy, or medical laboratory); 2) social service (e.g., child, youth, family, elderly, or disability services); 3) preschool or daycare; 4) K-12 school; 5) other schools and instructional settings (e.g., college, university, professional, business, technical or trade school, driving school, test preparation, or tutoring); 6) first response (e.g., police or fire protection, or emergency relief services); 7) death care (e.g., funeral home, crematory, or cemetery); 8) correctional facility (e.g., jail, prison, detention center, or reformatory); 9) food and beverage store (e.g., grocery store, warehouse club, supercenters, convenience store, specialty food store, or bakery); 10) agriculture, forestry, fishing, or hunting; 11) food manufacturing facility (e.g., meat processing, produce packing, or food or beverage manufacturing); 12) nonfood manufacturing facility (e.g., metals, equipment and machinery, or electronics); 13) public transit (e.g., bus, commuter rail, subway, or school bus); 14) United States Postal Service; and 15) other. Essential worker groups who responded with 1, 2, and 7 were categorized as "essential health care," 3-5 were categorized as "school and childcare," 6 and 8-14 were categorized as "other frontline," and 15 were categorized as "other essential," and those who answered "no" to the first question were categorized as "not an essential worker." Nonessential could include both employed and unemployed persons.

999 Work or school requirement was assessed by the following question: "Does your work or school require you to get a COVID-19 vaccine?" Response options were "yes," "no," or "unemployed/not applicable." Responses for "no" and "not applicable" were combined into one category. vaccine administration data ${ }^{* * *}(3)$. For all analyses, statistical significance was defined as $\mathrm{p}<0.05$. This activity was reviewed by the CDC and was conducted consistent with applicable federal law and CDC policy. ${ }^{\dagger \dagger \dagger \dagger}$

Prevalence of report of a provider recommendation for COVID-19 vaccines among adults increased from 34.6\% during April 22-May 29 to 40.5\% during August 29September 25, 2021 (Table 1). From April 22-May 29 to August 29-September 25, report of provider recommendation ranged from $34.3 \%$ in HHS Region 10 to $42.7 \%$ in HHS Region 2 (Supplementary Table 1, https://stacks.cdc.gov/ $\mathrm{view} / \mathrm{cdc} / 112307)$. Report of a provider recommendation was more common among adults aged $\geq 65$ years $(44.2 \%)$ than among those aged $18-29$ years $(28.3 \%)$; those with more than a college degree $(45.6 \%)$ than among those with a high school education or less $(33.5 \%)$; adults with annual household income of $\geq \$ 75,000$ (39.8\%) compared with those below the U.S. poverty threshold (36.9\%); adults with health insurance (39.1\%) compared with those without insurance (24.7\%); adults who are essential health care workers (51.8\%) compared with those in other essential work settings $(32.1 \%-38.8 \%)$; and adults with comorbidities (50.4\%) compared with those without (32.1\%) (Table 1).

Adults who had received a provider recommendation were more likely to have received $\geq 1$ dose of COVID-19 vaccine (77.6\%) than were those who did not receive a recommendation $(61.9 \%)(\mathrm{aPR}=1.12)$ (Table 2). Analyses of the interaction between provider recommendation and sociodemographic characteristics on vaccine receipt found that provider recommendation was associated with higher likelihood of receipt of $\geq 1$ COVID-19 vaccine dose among most subgroups, with highest aPR for younger adults (aged 18-29 and 30-39 years; $\mathrm{aPR}=1.22$ ), non-Hispanic American Indian or Alaska Native adults $(\mathrm{aPR}=1.19)$, adults living in rural areas $(\mathrm{aPR}=1.18)$, adults living in the West $(\mathrm{aPR}=1.17)$ or Midwest $(\mathrm{aPR}=1.15)$, and adults who did not have a school or work COVID-19 vaccination requirement $(\mathrm{aPR}=1.15)$.

Report of a provider recommendation was associated with concern about COVID-19 $(\mathrm{aPR}=1.31)$, confidence that COVID-19 vaccines are important to protect oneself $(\mathrm{aPR}=1.15)$, confidence that COVID-19 vaccination was very or completely safe $(\mathrm{aPR}=1.17)$, and perception that many or all of their family and friends had received COVID-19 vaccination $(\mathrm{aPR}=1.19)$ (Supplementary Table 2, https:// stacks.cdc.gov/view/cdc/112308).

\footnotetext{
**** Survey weights were calibrated to the COVID-19 vaccine administration data by jurisdiction, age group, and sex.

计计 45 C.F.R. part 46.102(l)(2), 21 C.F.R. part 56; 42 U.S.C. Sect. 241(d); 5 U.S.C. Sect. 552a; 44 U.S.C. Sect. 3501 et seq.
} 
TABLE 1. Characteristics of adults who reported a health care provider recommendation for COVID-19 vaccination, by selected sociodemographic characteristics and associated factors - National Immunization Survey-Adult COVID Module, United States, April 22-September 25, 2021

\begin{tabular}{|c|c|c|c|c|c|}
\hline \multirow[b]{3}{*}{ Characteristic } & \multirow{2}{*}{\multicolumn{2}{|c|}{ Overall }} & \multicolumn{3}{|c|}{ Provider recommendation } \\
\hline & & & \multirow{2}{*}{$\begin{array}{l}\text { Prevalence } \\
\%(95 \% \mathrm{Cl})\end{array}$} & \multicolumn{2}{|c|}{ Prevalence ratio } \\
\hline & No. & $\%(95 \% \mathrm{Cl})$ & & $\begin{array}{l}\text { Unadjusted } \\
(95 \% \mathrm{CI})\end{array}$ & $\begin{array}{c}\text { Adjusted* } \\
(95 \% \mathrm{Cl})\end{array}$ \\
\hline All adults & 340,543 & 100.0 & $37.4(37.1-37.7)$ & - & - \\
\hline \multicolumn{6}{|l|}{ Period of data collection } \\
\hline Apr 22-May 29 & 77,162 & $20.0(19.7-20.3)$ & $34.6(33.9-35.3)$ & Ref & Ref \\
\hline May 30-Jun 26 & 56,749 & $20.0(19.7-20.3)$ & $35.8(35.0-36.6)$ & $1.03(1.00-1.07)$ & $1.03(1.00-1.06)$ \\
\hline Jun 27-Jul 31 & 73,512 & $20.0(19.7-20.3)$ & $37.6(36.9-38.3)$ & $1.09(1.06-1.12)$ & $1.08(1.05-1.11)$ \\
\hline Aug 1-Aug 28 & 63,193 & $20.0(19.7-20.3)$ & $38.6(37.9-39.4)$ & $1.12(1.09-1.15)$ & $1.10(1.07-1.13)$ \\
\hline Aug 29-Sep 25 & 73,426 & $20.0(19.7-20.2)$ & $40.5(39.8-41.2)$ & $1.17(1.14-1.20)$ & $1.14(1.10-1.17)$ \\
\hline \multicolumn{6}{|l|}{ Age group, yrs } \\
\hline $18-29$ & 58,464 & $21.0(20.7-21.3)$ & $28.3(27.6-29.0)$ & $0.64(0.62-0.66)$ & $0.72(0.69-0.74)$ \\
\hline $30-39$ & 56,584 & $17.3(17.1-17.6)$ & $34.9(34.2-35.7)$ & $0.79(0.77-0.81)$ & $0.83(0.80-0.85)$ \\
\hline $40-49$ & 52,694 & $16.0(15.7-16.2)$ & $37.9(37.1-38.7)$ & $0.86(0.83-0.88)$ & $0.86(0.83-0.89)$ \\
\hline $50-64$ & 95,399 & $24.5(24.2-24.8)$ & $41.0(40.4-41.7)$ & $0.93(0.91-0.95)$ & $0.92(0.89-0.94)$ \\
\hline$\geq 65$ & 75,147 & $21.2(20.9-21.5)$ & $44.2(43.5-45.0)$ & Ref & Ref \\
\hline \multicolumn{6}{|l|}{ Sex } \\
\hline Male & 168,106 & $48.4(48.1-48.8)$ & $34.4(34.0-34.9)$ & Ref & Ref \\
\hline Female & 173,190 & $51.6(51.2-51.9)$ & $40.3(39.9-40.8)$ & 1.17 (1.15-1.19) & 1.07 (1.05-1.09) \\
\hline \multicolumn{6}{|l|}{ Transgender } \\
\hline Yes & 13,287 & $4.5(4.4-4.7)$ & $36.5(34.9-38.1)$ & $0.98(0.93-1.02)$ & $1.01(0.96-1.05)$ \\
\hline No & 309,379 & 95.5 (95.3-95.6) & $37.4(37.0-37.7)$ & Ref & Ref \\
\hline \multicolumn{6}{|l|}{ Sexual orientation } \\
\hline Heterosexual & 298,486 & $92.6(92.4-92.7)$ & $37.5(37.2-37.8)$ & Ref & Ref \\
\hline Gay or lesbian & 8,857 & $2.3(2.2-2.4)$ & $41.0(39.0-43.1)$ & 1.09 (1.04-1.15) & $1.12(1.06-1.17)$ \\
\hline Bisexual & 9,745 & $3.3(3.1-3.4)$ & $34.1(32.3-35.9)$ & $0.91(0.86-0.96)$ & $1.02(0.96-1.07)$ \\
\hline Other & 5,654 & $1.9(1.8-2.0)$ & $36.3(33.8-38.8)$ & $0.97(0.90-1.04)$ & $1.06(0.98-1.14)$ \\
\hline \multicolumn{6}{|l|}{ Race/Ethnicity } \\
\hline White, non-Hispanic & 210,659 & $62.1(61.8-62.4)$ & $37.3(36.9-37.7)$ & Ref & Ref \\
\hline Black, non-Hispanic & 40,610 & $12.0(11.8-12.2)$ & $38.4(37.5-39.4)$ & $1.03(1.00-1.06)$ & $1.02(0.99-1.05)$ \\
\hline Hispanic & 43,420 & $17.2(16.9-17.5)$ & $37.2(36.3-38.1)$ & $1.00(0.97-1.02)$ & $1.09(1.06-1.12)$ \\
\hline Asian, non-Hispanic & 17,859 & $4.2(4.1-4.3)$ & $40.0(38.5-41.6)$ & 1.07 (1.03-1.12) & $1.11(1.06-1.16)$ \\
\hline American Indian or Alaska Native, non-Hispanic & 8,319 & $1.3(1.3-1.4)$ & $38.8(36.2-41.5)$ & $1.04(0.97-1.12)$ & $1.13(1.05-1.21)$ \\
\hline Other or multiple races, non-Hispanic & 12,865 & $3.2(3.0-3.3)$ & $36.2(34.4-38.1)$ & $0.97(0.92-1.02)$ & $1.03(0.98-1.09)$ \\
\hline \multicolumn{6}{|l|}{ Educational level } \\
\hline High school or less & 85,450 & $39.1(38.7-39.4)$ & $33.5(33.0-34.1)$ & $0.74(0.72-0.75)$ & $0.83(0.80-0.85)$ \\
\hline Some college & 94,461 & $30.5(30.2-30.9)$ & $37.6(37.0-38.2)$ & $0.82(0.80-0.84)$ & $0.88(0.86-0.90)$ \\
\hline College graduate & 85,631 & $19.2(18.9-19.4)$ & $40.7(40.1-41.4)$ & $0.89(0.87-0.91)$ & $0.96(0.94-0.99)$ \\
\hline Above college graduate & 68,286 & $11.2(11.1-11.4)$ & $45.6(44.8-46.4)$ & Ref & Ref \\
\hline \multicolumn{6}{|l|}{ Annual household income, ${ }^{\dagger}$ USD } \\
\hline Below poverty & 32,552 & $11.3(11.1-11.5)$ & $36.9(35.9-37.9)$ & $0.93(0.90-0.95)$ & $1.00(0.96-1.03)$ \\
\hline Above poverty and $<\$ 75,000$ & 106,976 & $32.1(31.8-32.5)$ & $36.1(35.5-36.7)$ & $0.91(0.89-0.93)$ & $0.95(0.93-0.97)$ \\
\hline Above poverty and $\geq \$ 75,000$ & 129,250 & $32.7(32.4-33.0)$ & $39.8(39.3-40.3)$ & Ref & Ref \\
\hline Unknown income & 75,264 & $23.9(23.6-24.2)$ & $36.1(35.5-36.8)$ & $0.91(0.89-0.93)$ & $0.95(0.92-0.97)$ \\
\hline
\end{tabular}

See table footnotes on the next page.

In the jurisdiction-level correlation analysis, COVID-19 vaccination coverage was higher among persons living in jurisdictions with higher prevalence of provider recommendation (correlation coefficient $=0.66$ ) (Figure) (Supplementary Table 1, https://stacks.cdc.gov/view/cdc/112307). For example, in Wyoming, prevalence of report of a provider recommendation was $30.1 \%$, and COVID-19 vaccination coverage was $51.2 \%$, whereas in Puerto Rico, prevalence of provider recommendation was 50.5\%, and COVID-19 vaccination coverage was $77.5 \%$.

\section{Discussion}

Health care providers are among the most trusted sources of information on safety and effectiveness of vaccines, and their recommendations are strongly associated with vaccination acceptance $(4,5)$. This study found that provider recommendation was associated with higher likelihood of getting vaccinated, as well as higher likelihood of having concerns about COVID-19, confidence that vaccines are important to protect oneself from COVID-19, confidence that COVID-19 vaccines are very or completely safe, and perception that many 
TABLE 1. (Continued) Characteristics of adults who reported a health care provider recommendation for COVID-19 vaccination, by selected sociodemographic characteristics and associated factors - National Immunization Survey-Adult COVID Module, United States, April 22-September 25, 2021

\begin{tabular}{|c|c|c|c|c|c|}
\hline \multirow[b]{3}{*}{ Characteristic } & \multirow{2}{*}{\multicolumn{2}{|c|}{ Overall }} & \multicolumn{3}{|c|}{ Provider recommendation } \\
\hline & & & \multirow{2}{*}{$\begin{array}{l}\text { Prevalence } \\
\%(95 \% \mathrm{Cl})\end{array}$} & \multicolumn{2}{|c|}{ Prevalence ratio } \\
\hline & No. & $\%(95 \% \mathrm{Cl})$ & & $\begin{array}{c}\text { Unadjusted } \\
(95 \% \mathrm{Cl})\end{array}$ & $\begin{array}{l}\text { Adjusted* } \\
(95 \% \mathrm{Cl})\end{array}$ \\
\hline \multicolumn{6}{|l|}{ Health insurance status } \\
\hline Insured & 306,694 & $89.5(89.3-89.7)$ & $39.1(38.7-39.4)$ & Ref & Ref \\
\hline Not insured & 27,335 & $10.5(10.3-10.7)$ & $24.7(23.8-25.7)$ & $0.63(0.61-0.66)$ & $0.75(0.72-0.78)$ \\
\hline \multicolumn{6}{|l|}{ Essential worker status ${ }^{\S}$} \\
\hline Essential health care & 36,028 & $9.1(8.9-9.3)$ & $51.8(50.8-52.9)$ & $1.40(1.37-1.43)$ & $1.38(1.35-1.42)$ \\
\hline School and child care & 12,789 & $2.9(2.8-3.0)$ & $38.8(37.1-40.5)$ & $1.05(1.00-1.09)$ & $1.02(0.97-1.07)$ \\
\hline Other frontline & 24,835 & $8.4(8.2-8.6)$ & $32.3(31.2-33.4)$ & $0.87(0.84-0.90)$ & $1.02(0.98-1.06)$ \\
\hline Other essential & 39,597 & $12.5(12.2-12.7)$ & $32.1(31.2-33.0)$ & $0.87(0.84-0.89)$ & $1.00(0.97-1.04)$ \\
\hline Not an essential worker & 228,472 & $67.2(66.9-67.5)$ & $37.1(36.7-37.5)$ & Ref & Ref \\
\hline \multicolumn{6}{|l|}{ MSA } \\
\hline MSA, principal city & 106,173 & $29.1(28.8-29.4)$ & $38.6(38.0-39.2)$ & Ref & Ref \\
\hline MSA, nonprincipal city & 172,259 & $57.2(56.9-57.5)$ & $37.4(37.0-37.8)$ & $0.97(0.95-0.99)$ & $0.96(0.94-0.98)$ \\
\hline Non-MSA & 65,610 & $13.7(13.5-13.9)$ & $34.9(34.1-35.7)$ & $0.90(0.88-0.93)$ & $0.92(0.89-0.95)$ \\
\hline \multicolumn{6}{|l|}{ U.S. Census region } \\
\hline Northeast & 70,694 & $17.4(17.3-17.6)$ & $42.1(41.4-42.7)$ & Ref & Ref \\
\hline Midwest & 54,434 & $20.8(20.5-21.0)$ & $36.7(35.9-37.4)$ & $0.87(0.85-0.89)$ & $0.93(0.90-0.95)$ \\
\hline South & 70,212 & $23.8(23.5-24.0)$ & $36.3(35.6-37.1)$ & $0.86(0.84-0.89)$ & $0.88(0.86-0.91)$ \\
\hline West & 126,934 & $38.0(37.8-38.3)$ & $36.0(35.5-36.5)$ & $0.86(0.84-0.87)$ & $0.92(0.90-0.94)$ \\
\hline \multicolumn{6}{|l|}{ Comorbidities** } \\
\hline Yes & 102,135 & $29.2(28.9-29.5)$ & $50.4(49.8-51.1)$ & $1.57(1.54-1.60)$ & $1.47(1.44-1.50)$ \\
\hline No & 237,651 & $70.8(70.5-71.1)$ & $32.1(31.7-32.5)$ & Ref & Ref \\
\hline \multicolumn{6}{|l|}{ Disability status $^{\dagger \dagger}$} \\
\hline Yes & 30,864 & $9.7(9.5-9.9)$ & $44.9(43.8-46.0)$ & $1.23(1.20-1.26)$ & $1.11(1.07-1.14)$ \\
\hline No & 312,280 & $90.3(90.1-90.5)$ & $36.6(36.3-36.9)$ & Ref & Ref \\
\hline \multicolumn{6}{|c|}{ Work or school requirement ${ }^{\S \S}$} \\
\hline Yes & 43,949 & $10.8(10.6-11.0)$ & $49.8(48.8-50.7)$ & $1.39(1.36-1.42)$ & $1.32(1.29-1.36)$ \\
\hline No/Other & 297,453 & $89.2(89.0-89.4)$ & $35.9(35.5-36.2)$ & Ref & Ref \\
\hline
\end{tabular}

Abbreviations: $\mathrm{MSA}=$ metropolitan statistical area; Ref = referent group; USD = U.S. dollars.

* Adjusted for age group, sex, transgender identity, sexual orientation, race/ethnicity, education, income, insurance status, MSA, U.S. Census region, comorbidity status, disability status, and essential worker status.

† Household income is derived from the number of persons reported in the household, the reported household income, and the 2020 U.S. Census poverty thresholds.

$\S$ Essential worker status was defined based on the following questions: "Are you a frontline or essential worker according to your state or region?" and "In what location or setting do you currently work?" Essential worker groups were categorized as "essential healthcare,"'school and childcare,"'other frontline,"'"other essential," and "nonessential." Nonessential could include both employed and unemployed individuals.

" MSA status was determined based on household reported city and county of residence and was grouped into three categories: MSA principal city (urban), MSA nonprincipal city (suburban), and non-MSA (rural). MSAs and principal cities were as defined by the U.S. Census Bureau (https://www.census.gov/programs-surveys/ metro-micro.html). Non-MSA areas include urban populations not located within an MSA as well as completely rural areas.

** Comorbidity status was ascertained by the following question: "Do you have a health condition that may put you at higher risk for COVID-19?"

†+ Disability status was ascertained by the following question:"Do you have serious difficulty seeing, hearing, walking, remembering, making decisions, or communicating?"

$\S \S$ Work or school requirement was assessed by the following question: "Does your work or school require you to get a COVID-19 vaccine?" Response options were yes, no, or unemployed/not applicable. Responses for "no" and "not applicable" were combined into one category.

or all of one's family and friends had received COVID-19 vaccine. The findings from an ecologic analysis also suggest that jurisdictions' prevalence of provider recommendations was positively associated with jurisdiction-level COVID-19 vaccination coverage.

Similar to report of a provider recommendation for influenza vaccine, which was $33.0 \%$ in 2016 (6), report of a provider recommendation for vaccination against COVID-19 remains low. Approximately less than one half of participants nationwide reported receiving a provider recommendation, with $<40 \%$ of persons in rural areas and in some jurisdictions reporting a provider recommendation. These patterns mirror known patterns in disparities in health insurance coverage, financial barriers to care, and the use of wellness visits and checkups; as a result, lower access to health care might reduce the opportunity for interactions with trusted providers (7).

As COVID-19 vaccine availability in primary care settings increases and patients become eligible for additional or booster doses, provider recommendation will continue to serve an important role in motivating individual patient vaccination acceptance and completion (8). Health care systems and medical practices can benefit from procedures that build 
TABLE 2. Association of report of a health care provider recommendation for COVID-19 vaccination and receipt of $\geq 1$ COVID-19 vaccine dose, overall and by selected sociodemographic characteristics - National Immunization Survey-Adult COVID Module, United States, April 22September 25,2021

\begin{tabular}{|c|c|c|c|c|}
\hline \multirow[b]{3}{*}{ Characteristic } & \multicolumn{4}{|c|}{ Receipt of $\geq 1$ COVID-19 vaccine dose, $\%(95 \% \mathrm{Cl})$} \\
\hline & \multicolumn{2}{|c|}{ Provider recommendation } & \multicolumn{2}{|c|}{ Prevalence ratio } \\
\hline & Yes & No & Unadjusted & Adjusted* \\
\hline Overall & $77.6(77.1-78.1)$ & $61.9(61.5-62.3)$ & $1.25(1.24-1.27)$ & $1.12(1.11-1.14)$ \\
\hline \multicolumn{5}{|l|}{ Age group, yrs } \\
\hline $18-29$ & $63.3(61.9-64.7)$ & $45.4(44.5-46.3)$ & $1.39(1.35-1.44)$ & $1.22(1.18-1.26)$ \\
\hline $30-39$ & $68.7(67.4-70.1)$ & $51.1(50.1-52.2)$ & $1.34(1.31-1.38)$ & $1.22(1.18-1.25)$ \\
\hline $40-49$ & $74.5(73.2-75.8)$ & $57.5(56.4-58.6)$ & $1.30(1.26-1.33)$ & $1.19(1.16-1.22)$ \\
\hline $50-64$ & $80.9(80.0-81.8)$ & $70.0(69.1-70.8)$ & $1.16(1.14-1.18)$ & $1.08(1.06-1.10)$ \\
\hline$\geq 65$ & $91.0(90.3-91.7)$ & $87.4(86.7-88.1)$ & $1.04(1.03-1.05)$ & $1.03(1.01-1.04)$ \\
\hline \multicolumn{5}{|l|}{ Sex } \\
\hline Male & $78.9(78.2-79.5)$ & $64.2(63.6-64.9)$ & $1.23(1.21-1.24)$ & $1.12(1.11-1.14)$ \\
\hline Female & $78.9(78.2-79.5)$ & $64.2(63.6-64.9)$ & $1.23(1.21-1.24)$ & $1.15(1.13-1.16)$ \\
\hline \multicolumn{5}{|l|}{ Race/Ethnicity } \\
\hline White, non-Hispanic & $80.5(79.9-81.1)$ & $63.1(62.6-63.7)$ & $1.27(1.26-1.29)$ & $1.17(1.15-1.19)$ \\
\hline Black, non-Hispanic & $69.0(67.5-70.5)$ & $56.4(55.1-57.7)$ & $1.22(1.19-1.26)$ & $1.07(1.03-1.10)$ \\
\hline Hispanic & $74.0(72.6-75.4)$ & $60.2(58.9-61.4)$ & $1.23(1.20-1.26)$ & $1.09(1.06-1.12)$ \\
\hline Asian, non-Hispanic & $88.3(86.2-90.5)$ & $87.1(85.5-88.7)$ & $1.01(0.98-1.05)$ & $1.01(0.96-1.05)$ \\
\hline American Indian or Alaska Native, non-Hispanic & $69.1(64.7-73.5)$ & $46.6(43.1-50.1)$ & $1.48(1.34-1.64)$ & $1.19(1.09-1.30)$ \\
\hline Other or multiple races, non-Hispanic & $67.8(64.6-71.1)$ & $48.7(46.3-51.1)$ & $1.39(1.30-1.49)$ & $1.15(1.07-1.24)$ \\
\hline \multicolumn{5}{|l|}{ Essential worker ${ }^{\dagger}$} \\
\hline Essential health care & $81.8(80.6-83.1)$ & $68.3(66.7-69.8)$ & $1.20(1.17-1.23)$ & $1.12(1.08-1.15)$ \\
\hline School and child care & $84.9(82.4-87.4)$ & $78.5(76.4-80.5)$ & $1.08(1.04-1.13)$ & $1.04(0.99-1.10)$ \\
\hline Other frontline & $67.0(64.8-69.1)$ & $52.2(50.7-53.7)$ & $1.28(1.23-1.34)$ & $1.10(1.07-1.14)$ \\
\hline Other essential & $68.2(66.5-69.9)$ & $50.2(49.0-51.4)$ & $1.36(1.31-1.41)$ & $1.15(1.11-1.18)$ \\
\hline Not an essential worker & $79.3(78.7-79.9)$ & $64.5(63.9-65.0)$ & $1.23(1.22-1.24)$ & $1.15(1.13-1.16)$ \\
\hline \multicolumn{5}{|l|}{$M S A^{\S}$} \\
\hline MSA, principal city & 78.1 (77.2-79.0) & $65.4(64.6-66.2)$ & $1.19(1.17-1.21)$ & $1.10(1.08-1.12)$ \\
\hline MSA, nonprincipal city & $78.6(78.0-79.3)$ & $62.6(62.0-63.2)$ & $1.26(1.24-1.27)$ & $1.14(1.12-1.16)$ \\
\hline Non-MSA & 71.7 (70.3-73.0) & $52.2(51.1-53.3)$ & $1.37(1.33-1.41)$ & $1.18(1.14-1.21)$ \\
\hline \multicolumn{5}{|l|}{ U.S. Census region } \\
\hline Northeast & $81.8(80.9-82.8)$ & $71.9(71.0-72.8)$ & $1.14(1.12-1.16)$ & $1.08(1.05-1.10)$ \\
\hline Midwest & $75.4(74.2-76.6)$ & $57.9(56.9-59.0)$ & $1.30(1.27-1.33)$ & $1.15(1.12-1.18)$ \\
\hline South & $81.3(80.2-82.4)$ & $67.8(66.8-68.8)$ & $1.20(1.18-1.22)$ & $1.11(1.09-1.14)$ \\
\hline West & 74.1 (73.3-74.9) & $55.9(55.3-56.6)$ & $1.32(1.30-1.35)$ & $1.17(1.15-1.19)$ \\
\hline \multicolumn{5}{|l|}{ Comorbidities" } \\
\hline Yes & $83.5(82.8-84.2)$ & $71.2(70.3-72.1)$ & $1.17(1.15-1.19)$ & $1.15(1.13-1.17)$ \\
\hline No & $74.1(73.4-74.7)$ & $59.3(58.8-59.8)$ & $1.25(1.23-1.27)$ & $1.13(1.11-1.14)$ \\
\hline \multicolumn{5}{|l|}{ Work or school requirement ${ }^{* *}$} \\
\hline Yes & $88.2(87.1-89.2)$ & $85.7(84.6-86.8)$ & $1.03(1.01-1.05)$ & $1.03(1.01-1.05)$ \\
\hline No/Other & $75.8(75.2-76.3)$ & $59.6(59.2-60.1)$ & $1.27(1.26-1.28)$ & $1.15(1.14-1.17)$ \\
\hline
\end{tabular}

Abbreviation: MSA = metropolitan statistical area

* Adjusted for age group, sex, transgender identity, sexual orientation, race/ethnicity, education, income, insurance status, MSA, U.S. Census region, comorbidity status, disability status, and essential worker status.

† Essential worker status was defined based on the following questions: "Are you a frontline or essential worker according to your state or region?" and "In what location or setting do you currently work?" Essential worker groups were categorized as "essential healthcare,"'school and childcare,"'"other frontline,"'other essential," and "nonessential." Nonessential may include both employed and unemployed individuals.

$\S$ MSA status was determined based on household reported city and county of residence and was grouped into three categories: MSA principal city (urban), MSA nonprincipal city (suburban), and non-MSA (rural). MSAs and principal cities were as defined by the U.S. Census Bureau (https://www.census.gov/programs-surveys/ metro-micro.html). Non-MSA areas include urban populations not located within an MSA as well as completely rural areas.

" Comorbidity status was ascertained by the following question: "Do you have a health condition that may put you at higher risk for COVID-19?"

** Work or school requirement was assessed by the following question: "Does your work or school require you to get a COVID-19 vaccine?" Response options were yes, no, or unemployed/not applicable. Responses for "no" and "not applicable" were combined into one category.

patient and provider confidence in COVID-19 vaccination and strengthen the capacity of health care providers to have conversations about vaccines, address misinformation, and provide tailored information to patients. As trusted sources of medical information, providers have the opportunity to clearly recommend COVID-19 vaccines as a main strategy for preventing serious health outcomes from COVID-19 (9). 
FIGURE. Correlation of prevalence of report of health care provider recommendation and COVID-19 vaccination coverage ( $\geq 1$ dose) among 53 jurisdictions, * by jurisdiction - National Immunization Survey Adult-COVID Module, United States, April 22-September 25, 2021

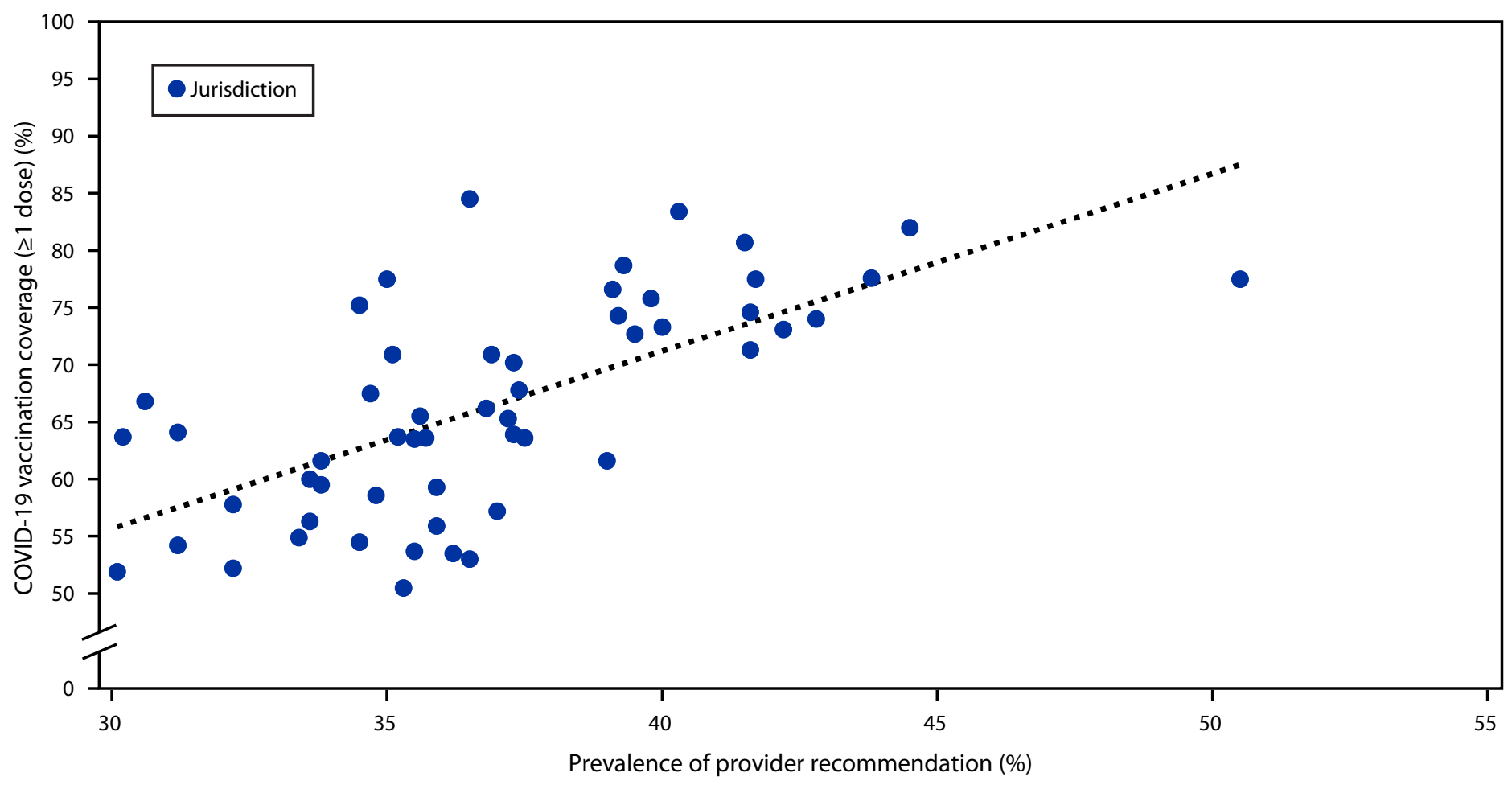

* Sample correlation coefficient $=0.66$.

The findings in this study are subject at least six limitations. First, response rates were low (approximately 20\%), but consistent with other NIS surveys (2). Bias in estimates might remain after weighting for household nonresponse and incomplete sample frame (households with only landline or no telephone service were excluded). Second, vaccination receipt, provider recommendation, and other characteristics (e.g., essential worker status or medical conditions) were selfreported and subject to recall and misclassification bias. For example, the question on medical conditions could have been interpreted by some survey respondents as medical conditions that place them at higher risk for exposure to COVID-19; however, a secondary analysis of a follow-up question on condition type found that approximately $75 \%$ indicated a medical condition associated with higher risk for severe COVID-19. Moreover, survey weights were calibrated to COVID-19 vaccine administration data (3) to mitigate possible bias from incomplete sample frame, nonresponse, and misclassification of vaccination status. Third, the survey did not measure health care provider visits, so a low number of reports of provider recommendation could be due to limited access to health care providers. Fourth, attitudes might have changed over time with changes in the Advisory Committee on Immunization Practices

\section{Summary \\ What is already known about this topic? \\ COVID-19 vaccination is critical to controlling the COVID-19 pandemic; health care providers play an important role in achieving high vaccination coverage. \\ What is added by this report? \\ Adults who reported a provider COVID-19 vaccination recom- mendation were more likely to have been vaccinated, to be concerned about COVID-19, to have confidence that COVID-19 vaccines are important and safe, and to perceive that family and friends had been vaccinated. \\ What are the implications for public health practice? \\ A health care provider recommendation for COVID-19 vaccines at every visit could increase coverage and confidence in vaccines, particularly among groups with lower COVID-19 vaccination coverage, including younger adults, racial/ethnic minorities, and rural residents.}

vaccination recommendations or the emergence of the highly transmissible SARS-CoV-2 B.1.617.2 (Delta) variant (10). Fifth, the categorization of attitudinal measures was conservative (e.g., classifying someone who reported "somewhat safe" as not believing COVID-19 vaccination is safe), which might 
have underestimated observed associations. Finally, the survey is cross-sectional; thus, causal relationships cannot be inferred, including the association between beliefs about COVID-19 vaccination and report of a provider recommendation. For example, providers might be more likely to recommend vaccines to persons who express more concerns or who seem more receptive to vaccination; alternatively, these persons might be more likely to remember and report receiving a provider recommendation. In addition, causality between the ecological association of provider recommendation at the jurisdiction level and vaccination coverage cannot be inferred.

Health care providers are uniquely positioned to provide COVID-19 vaccination recommendations, and it is important that they continue to promote COVID-19 vaccination to eligible persons. This is particularly important among groups with lower COVID-19 vaccination coverage, including younger adults, racial/ethnic minorities, persons with lower education and income, and rural residents. Empowering health care providers to recommend COVID-19 vaccines at every visit and reducing barriers to health care access could increase confidence in vaccines and COVID-19 vaccination coverage.

Corresponding author: Kimberly H. Nguyen, knguyen2@cdc.gov.

${ }^{1}$ CDC COVID-19 Vaccine Task Force; ${ }^{2}$ Department of Health Behavior, Gillings School of Global Public Health, and Lineberger Comprehensive Cancer Center, University of North Carolina-Chapel Hill, Chapel Hill, North Carolina.

All authors have completed and submitted the International Committee of Medical Journal Editors form for disclosure of potential conflicts of interest. Noel T. Brewer reports consulting fees from Merck and Novartis. No other potential conflicts of interest were disclosed.

\section{References}

1. CDC. COVID-19 vaccination field guide: 12 strategies for your community. Atlanta, GA: US Department of Health and Human Services, CDC; 2021. https://www.cdc.gov/vaccines/covid-19/ downloads/vaccination-strategies.pdf

2. CDC; NORC. National Immunization Survey-Child: a user's guide for the 2019 public-use data file. Atlanta, GA: US Department of Health and Human Services, CDC; 2021. https://www.cdc.gov/vaccines/imzmanagers/nis/downloads/NIS-PUF19-DUG.pdf

3. CDC. COVID data tracker. Atlanta, GA: US Department of Health and Human Services, CDC; 2021. https://covid.cdc.gov/ covid-data-tracker/

4. Oh NL, Biddell CB, Rhodes BE, Brewer NT. Provider communication and HPV vaccine uptake: a meta-analysis and systematic review. Prev Med 2021;148:106554. PMID:33857561 https://doi.org/10.1016/j. ypmed.2021.106554

5. Opel DJ, Mangione-Smith R, Robinson JD, et al. The influence of provider communication behaviors on parental vaccine acceptance and visit experience. Am J Public Health 2015;105:1998-2004. PMID:25790386 https://doi.org/10.2105/AJPH.2014.302425

6. Lu PJ, Srivastav A, Amaya A, et al. Association of provider recommendation and offer and influenza vaccination among adults aged $\geq 18$ years-United States. Vaccine 2018;36:890-8. PMID:29329685 https://doi.org/10.1016/j.vaccine.2017.12.016

7. Derose KP, Gresenz CR, Ringel JS. Understanding disparities in health care access - and reducing them-through a focus on public health. Health Aff (Millwood) 2011;30:1844-51. PMID:21976325 https:// doi.org/10.1377/hlthaff.2011.0644

8. CDC. Expanding COVID-19 vaccine distribution to primary care providers to address disparities in immunization. Atlanta, GA: US Department of Health and Human Services, CDC; 2021. https://www. cdc.gov/vaccines/covid-19/downloads/Guide-for-Jurisdictions-on-PCPCOVID-19-Vaccination.pdf

9. CDC. Vaccines \& immunizations: talking with patients about COVID-19 vaccination. Atlanta, GA: US Department of Health and Human Services, CDC; 2021. https://www.cdc.gov/vaccines/covid-19/ hcp/engaging-patients.html

10. Mbaeyi S, Oliver SE, Collins JP, et al.; CDC. The advisory committee on immunization practices' interim recommendations for additional primary and booster doses of COVID-19 vaccines_-United States, 2021. MMWR Morb Mortal Wkly Rep 2021;70:1545-52. PMID:34735422 https://doi.org/10.15585/mmwr.mm7044e2 